\title{
Quadratic Pencils and Least-Squares Piecewise-Polynomial Approximation*
}

\author{
By Boris Mityagin
}

\begin{abstract}
For a partition $\xi=\left(0=\xi_{0}<\xi_{1}<\cdots<\xi_{n}<\xi_{n+1}=1\right)$ of the unit interval, $S_{\xi}^{k m}$, $k>m$, denotes the space of piecewise-polynomials of order $k$ and of smoothness $m-1$; this space can be represented as the graph of the appropriate linear operator between two finite-dimensional Hilbert spaces. It gives an approach to the C. de Boor problem, 1972, on uniform boundedness (with respect to $\xi$ ) in the $L_{\infty}$-norm of the orthogonal projections onto $S_{\xi}^{k m}$, and we give the detailed analysis of a quadratic pencil (matrix-valued polynomial of the second degree) which appears in the case of a geometric mesh $\xi$ if $2 m \leqslant k$. The explicit calculations and estimates of zeros of the "characteristic" polynomial show that in the case $S_{\xi(x)}^{63}, \xi(x)$ the geometric mesh with the parameter $x, 0<x<\infty$, the orthogonal projectors are uniformly bounded.
\end{abstract}

0. Introduction. For any pair of integers $k, m, k>m>0$, and any partition $\xi=\left(\xi_{\alpha}\right)_{0}^{n+1}$ of the unit interval $[0,1]$,

$$
0=\xi_{0}<\xi_{1}<\cdots<\xi_{n}<\xi_{n+1}=1,
$$

we define the piecewise-polynomial subspace

$$
S \equiv S_{\xi}^{k m}=\mathbf{P}_{k, \xi} \cap C^{(m-1)}[0,1]
$$

where

$$
\begin{gathered}
\mathbf{P}_{k, \xi}=\left\{f \in L[0,1]: f \mid \Delta_{\alpha} \text { is a polynomial } \sum_{0}^{k-1} c_{i}^{\alpha} x^{i} \text { of order } k, 0 \leqslant \alpha \leqslant n\right\}, \\
\Delta_{\alpha}=\left[\xi_{\alpha}, \xi_{\alpha+1}\right] .
\end{gathered}
$$

Each interior breakpoint $\xi_{\alpha}, 1 \leqslant \alpha \leqslant n$, generates $m$ continuity conditions

$$
\left(f \mid \Delta_{\alpha-1}\right)^{(i)}\left(\xi_{\alpha}\right)=\left(f \mid \Delta_{\alpha}\right)^{(i)}\left(\xi_{\alpha}\right), \quad 0 \leqslant i<m,
$$

to make a function $f \in \mathbf{P}_{k \xi}$ be an element of $S_{\xi}^{k m}$.

Let $Q=Q_{S}$ be the orthogonal projection onto $S$ in $L^{2}[0,1]$, i.e. with respect to the inner product

$$
(f, g)=\int_{0}^{1} f(x) \overline{g(x)} d x .
$$

We are interested in $Q$ as a map in $C[0,1]$ or $L^{\infty}[0,1]$ and we would like to get the estimates of its norm

$$
\|Q\|_{\infty}=\sup _{f}\|Q f\|_{\infty} /\|f\|_{\infty}
$$

where $\|f\|_{\infty}=$ ess $\sup _{0 \leqslant x \leqslant 1}|f(x)|, f \in L^{\infty}[0,1]$.

Received September 29, 1980.

1980 Mathematics Subject Classification. Primary 41A15; Secondary 47A68.

* This paper was partly supported by NSF grant 7906079. 
The general problem (C. de Boor, 1972) on uniform boundedness of these projectors in $L^{\infty}$ over any partition $\xi$ (for fixed $k$ and $m$ ) remains unsolved. The series of important particular cases was treated by Z. Ciesielski [1], C. de Boor [2], J. Douglas, T. Dupont and L. Wahlbin [5], S. Demko [6] and others [11].

We now consider the case $k \geqslant 2 m$. It seems that with respect to the smoothness conditions this case is a degenerate one; however, for our approach (see Section 1 below) this is the only nondegenerate case. We represent the space $S=S_{\xi}^{k m}$ as the graph of a certain linear operator $l$ between two Hilbert (finite-dimensional) spaces so the projections can be written explicitly in terms of $l$ and $l^{*}$. In the case of the geometric mesh, i.e.

$$
\mu_{\alpha}:=\frac{\Delta_{\alpha+1}}{\Delta_{\alpha}}=\frac{\xi_{\alpha+2}-\xi_{\alpha+1}}{\xi_{\alpha+1}-\xi_{\alpha}} \equiv \mu \quad \text { for any } \alpha, 0 \leqslant \alpha<n,
$$

this construction produces $(m \times m)$-block Toeplitz operators, and the analysis of the corresponding quadratic pencil (see [7], [8]) is the crucial point to get the estimates of norms of $Q_{s}$. In particular, we prove that least-squares approximants by quintic splines on a geometric mesh with triple knots (the case $\mathbf{P}_{\xi}^{6,3}, \boldsymbol{\xi}$ of $(0,1)$ ) are uniformly bounded in $L^{\infty}$-, or $C$-, norm independently on the mesh-ratio $\mu$ in (0.4) or the number $n$ of breakpoints in $(0.1)$.

I want to thank Professor C. de Boor and Professor S. Demko who directed my attention to the problem and told me of previous results and Professor I. Gohberg for discussions on factorization of holomorphic operator-valued functions.

I started this work in the Summer of 1979 during my visit to Bonn University, SFB-72. I appreciate the help and support of SFB-72, and the nice atmosphere of the Institute that I felt very much, in particular, in my contacts with Professor H. Unger, Professor H. König and Professor K. Scherer.

1. Reduction of the Problem to an Analysis of a Finite-Dimensional VectorSequence Space. For the partition ( 0.1$)$ let us define for any $\alpha, 0 \leqslant \alpha \leqslant n$

$$
\varphi_{\alpha}(t)= \begin{cases}0, & t=\xi_{\alpha}, \\ 1, & t=\xi_{\alpha+1} \\ \text { linear on } \Delta_{\alpha}, & \end{cases}
$$

so $\varphi_{\alpha}: \Delta_{\alpha} \rightarrow I$, and for any function $f \in L_{1}[0,1]$ we put $\Phi_{\xi} f=\left(f_{\alpha}\right)_{0}^{n}, f_{\alpha} \in L_{1}[0,1]$, $f_{\alpha}(x)=f\left(\varphi_{\alpha}^{-1}(x)\right)$. This operator $\Phi_{\xi}$ maps $L^{2}[0,1]$ isometrically onto $l_{n+1}^{2}\left(L^{2}(I), \Delta\right)$, the Hilbert space of sets $g=\left(g_{\alpha}\right)_{0}^{n}, g_{\alpha} \in L^{2}(I)$, with the inner product

$$
(g, h)=\sum_{\alpha=0}^{n} \Delta_{\alpha} \cdot \int_{0}^{1} g(x) \overline{h(x)} d x,
$$

and the same operator $\Phi_{\xi}$ gives the isometrical isomorphism

$$
\begin{aligned}
L^{\infty}[0,1] & \stackrel{\sim}{\rightarrow} l_{n+1}^{\infty}\left(L^{\infty}[0,1]\right) \\
& =\text { the Banach space of }(n+1) \text {-triples } g=\left(g_{\alpha}\right)_{0}^{n}, g_{\alpha} \in L^{\infty}(I)
\end{aligned}
$$

with the norm

$$
\|g\|=\max _{0 \leqslant \alpha \leqslant n} \operatorname{ess} \sup _{x \in I}-\left|g_{\alpha}(x)\right|
$$


Remark. This cutting does not work if we consider the spaces of continuous functions, i.e. $\Phi_{\xi}(C(I))$ is a proper subspace of $l_{n+1}^{\infty}(C(I))$, of codimension $n$. But we do not need the special analysis in $C$ because the norm of the projection $Q_{S}$ is the same in $L_{\infty}$ and $C$ by the general argument: $S$ is a finite-dimensional subspace of continuous functions so $Q_{S}$ is an integral operator $f \rightarrow \int_{0}^{1}\left(\sum_{1}^{d} h_{i}(x) h_{i}(y)\right) f(y) d y$, where $\left(h_{i}\right)_{1}^{d}$ is an orthonormal basis in $S$ and the continuity of these function $h$ 's and the Lusin theorem imply that

$$
\left\|Q: L_{\infty} \rightarrow L_{\infty}\right\|=\|Q: C \rightarrow C\| .
$$

What is the image of $S_{\xi}$ (see (0.2) and (0.3)) under the mapping $\Phi_{\xi}$ ? The continuity conditions (0.3) after the isomorphism $\Phi_{\xi}$ have the following form:

$$
\Delta_{\alpha}^{-i} g_{\alpha}^{(i)}(1)=\Delta_{\alpha+1}^{-i} g_{\alpha+1}^{(i)}(0), \quad 0 \leqslant i<m, 0 \leqslant \alpha \leqslant n .
$$

(Recall that $g_{\alpha}(x)=\left(f \mid \Delta_{\alpha}\right)\left(\xi_{\alpha}+x\left(\xi_{\alpha+1}-\xi_{\alpha}\right)\right)$.) Instead of the projection $Q=Q_{S}$ in the original spaces $L^{2}(I)$ or $L^{\infty}(I)$ we have to analyze the orthogonal projection $\tilde{Q}: l_{n+1}^{2}\left(L^{2}(I), \Delta\right) \rightarrow \tilde{S}=\left\{g=\left(g_{\alpha}\right)_{0}^{n}: g_{\alpha}\right.$ are polynomials of $P_{k}$ and (1.1) holds $\}$ as an operator in $l_{n+1}^{\infty}\left(L^{\infty}(I)\right)$.

These spaces are infinite-dimensional but instead of the entire space $L^{2}(I)$, or $L^{\infty}(I)$, as a coordinate one, we can consider only polynomials $P_{k}$; more precisely, for (any) fixed $\alpha$

$$
L^{2}(I)=P_{k} \oplus P_{k}^{\perp}
$$

so

$$
l_{n+1}^{2}\left(L^{2}(I) ; \Delta\right)=l_{n+1}^{2}\left(P_{k} ; \Delta\right) \oplus l_{n+1}^{2}\left(P_{k}^{\perp} ; \Delta\right)
$$

and if $p_{k}: L^{2}(I) \rightarrow P_{k}$ is the orthogonal projection onto the subspace of polynomials of order $k$ and

$$
\tilde{p}_{k}^{n}: l_{n+1}^{2}\left(L^{2}(I) ; \Delta\right) \rightarrow l_{n+1}^{2}\left(P_{k} ; \Delta\right)
$$

is the orthogonal projection generated by the decomposition (1.2), (1.3), then

$$
\begin{aligned}
\left\|\tilde{p}_{k}^{n}\right\|_{\infty} & =\left\|\tilde{p}_{k}^{n}: l_{n+1}^{\infty}\left(L^{\infty}(I)\right) \rightarrow l_{n+1}^{\infty}\left(P_{k}\right)\right\| \\
& =\left\|p_{k}: L^{\infty}(I) \rightarrow P_{k}\right\|=\left\|p_{k}\right\|_{\infty}=C_{1}(k)<\infty,
\end{aligned}
$$

and this constant $C_{1}$ does not depend on the partition $\xi$, i.e. on the number $n$ of breakpoints and weights $\Delta=\left(\Delta_{\alpha}\right)_{0}^{n}$. The space $P_{k}=\left\{\sum_{0}^{k-1} c_{i} x^{i}\right\}$ is finite-dimensional and the norms $\|\cdot\|_{\infty}$ and $\|\cdot\|_{2}$ are equivalent:

$$
\|f\|_{2} \leqslant\|f\|_{\infty} \leqslant k^{1 / 2}\|f\|_{2} \quad \forall f \in P_{k} .
$$

For this reason we can consider the $L^{2}$-norm in the coordinate space $P_{k}$ in both the $l_{n+1}^{\infty}\left(P_{k}\right)$ - and $l_{n+1}^{2}\left(P_{k} ; \Delta\right)$-cases; it will change the $L^{\infty}$-norms of operators under consideration by some factors which are uniformly bounded with respect to $n$ and $\xi$. The piecewise-polynomial subspace $\tilde{S}_{\xi}$ is a subspace of $L=l_{n+1}^{2}\left(\mathbf{P}_{k} ; \Delta\right)$, or, what is the same, $l_{n+1}^{\infty}\left(\mathbf{P}_{k}\right)$, so $\tilde{Q}=(\tilde{Q} \mid L) \cdot \tilde{p}_{k}^{n}$ and

$$
\|\tilde{Q}\|_{\infty} \leqslant\|\tilde{Q} \mid L\|_{\infty} \cdot\left\|\tilde{P}_{k}^{n}\right\|_{\infty}=c_{1}(k)\left\|q^{\prime}\right\|_{\infty}
$$

by (1.4), where $q^{\prime}=\tilde{Q} \mid L$.

And we can consider the finite-dimensional space $L$ with two norms $l_{n+1}^{2}\left(\mathbf{P}_{k} ; \Delta\right)$ and $l_{n+1}^{\infty}\left(\mathbf{P}_{k}\right)$, and the projection $q^{\prime}$ only. 
2. Representation of $\tilde{S_{\xi}}$ as a Graph of a Linear Operator. For a given $m, 0 \leqslant$ $m<k, 2 m \leqslant k$, we split $\mathbf{P}_{k}$ by

$$
\mathbf{P}_{k}=A \oplus B, \quad B=\text { linear } \operatorname{span}\left\{x^{j} ; k-m \leqslant j<k\right\}
$$

and $A=\left\{f \in \mathbf{P}_{k}: f \perp B\right\}$.

We choose the special basis $\left\{e_{i}\right\}_{0}^{k-m-1}$ in $A$ :

$$
e_{i}=x^{i} / i !+\beta_{i}(x), \quad \beta_{i} \in B, 0 \leqslant i<k-m .
$$

Then

$$
e_{i}^{(j)}(0)=\delta_{i j}, \quad 0 \leqslant i, j<k-m
$$

Put

$$
s_{j i}=e_{i}^{(j)}(1)
$$

so

$$
S: \alpha \rightarrow c, \quad c_{j}=\sum_{0}^{k-m-1} s_{j i} a_{i}, \quad c=\sum_{0}^{m-1} c_{j} e_{j}
$$

is a mapping $A \rightarrow A$. For any $a=\sum \tilde{a}_{i} e_{i}$ and $a^{\prime}=\sum \tilde{a}_{i}^{\prime} e_{i}$ in $A$ their inner product is

$$
\left(a, a^{\prime}\right)=\left\langle T \tilde{a}, \tilde{a}^{\prime}\right\rangle, \quad \text { where } T=\left\{\left(e_{i}, e_{j}\right)\right\}_{0}^{k-m-1}
$$

is the Gramian of the basis $(2.2)$ and $\langle\cdot, \cdot\rangle$ is the canonical inner product in the coordinate $l^{2}$-space.

In the subspace $B$ we choose the basis $\left(v_{i}\right)_{0}^{m-1}$ in such a way that

$$
v_{i}^{(j)}(1)=\delta_{i j}, \quad 0 \leqslant i, j<m,
$$

and then $B$ is isometrically isomorphic to $l_{m}^{2}[V]$, i.e. for $b, b^{\prime} \in B, b=\sum_{0}^{m-1} \tilde{b}_{i} v_{i}(x)$,

$$
\left(b, b^{\prime}\right)=\left\langle V \tilde{b}, \tilde{b^{\prime}}\right\rangle, \quad \text { where } V=\left\{\left(v_{i}, v_{j}\right)\right\}_{0}^{m-1} \text {. }
$$

The results of explicit computation of these polynomials and matrices are presented below, $k=2 m$, for $m=1,2,3$.

(2.9.1) $m=1, e_{0}=1-3 / 2 x ; \quad v_{0}=x, S=-1 / 2 ; \quad T=1 / 4 ; \quad V=1 / 3$.

(2.9.2) $m=2$,

$$
\begin{gathered}
e_{0}=1-\frac{15}{2} x^{2}+7 x^{3} ; \quad v_{0}=3 x^{2}-2 x^{3} \\
e_{1}=x-3 x^{2}+\frac{21}{10} x^{3} ; \quad v_{1}=-x^{2}+x^{3} \\
S=\left[\begin{array}{cc}
1 / 2 & 1 / 10 \\
6 & 13 / 10
\end{array}\right], \quad T=\left[\begin{array}{cc}
1 / 4 & 1 / 40 \\
1 / 40 & 1 / 300
\end{array}\right], \quad V=\left[\begin{array}{cc}
13 / 35 & -11 / 210 \\
-11 / 210 & 1 / 105
\end{array}\right] .
\end{gathered}
$$

(2.9.3) $m=3$,

$$
\begin{aligned}
& e_{0}=1-42 x^{3}+90 x^{4}-\frac{99}{2} x^{5} \\
& e_{1}=x-\frac{72}{5} x^{3}+\frac{192}{7} x^{4}-\frac{99}{7} x^{5} \\
& e_{2}=\frac{1}{2} x^{2}-\frac{9}{4} x^{3}+\frac{45}{14} x^{4}-\frac{165}{112} x^{5}
\end{aligned}
$$




$$
\begin{gathered}
S=\left[\begin{array}{ccc}
-1 / 2 & -4 / 35 & -1 / 112 \\
-27 / 2 & -16 / 5 & -29 / 112 \\
-162 & -1404 / 35 & -95 / 28
\end{array}\right], \quad T=\left[\begin{array}{ccc}
1 / 4 & 1 / 35 & 1 / 672 \\
1 / 35 & 16 / 3675 & 1 / 3920 \\
1 / 672 & 1 / 3920 & 1 / 62720
\end{array}\right], \\
v_{0}=10 x^{3}-15 x^{4}+6 x^{5}, \\
v_{1}=-4 x^{3}+7 x^{4}-3 x^{5} \\
v_{2}=\frac{1}{2} x^{3}-x^{4}+\frac{1}{2} x^{5}, \\
V=\left[\begin{array}{ccc}
\frac{181}{462} & -\frac{311}{4620} & \frac{281}{55440} \\
-\frac{311}{4620} & \frac{52}{3465} & -\frac{23}{18480} \\
\frac{281}{55440} & -\frac{23}{18480} & \frac{1}{9240}
\end{array}\right] .
\end{gathered}
$$

Any element (or function) $f=\left(f_{\alpha}\right)_{0}^{n} \in L=l_{n+1}^{2}\left(\mathbf{P}_{k} ; \Delta\right)$ can be decomposed as $f=\left\{\left(a_{\alpha} ; b_{\alpha}\right)\right\}_{0}^{n}$, where $a_{\alpha}=\operatorname{Pr}_{A} f_{\alpha}, b_{\alpha}=\operatorname{Pr}_{B} f_{\alpha}, 0 \leqslant \alpha \leqslant n$, and $\operatorname{Pr}_{A}, \operatorname{Pr}_{B}$ are the orthogonal projectors to the first and second components in (2.1), correspondingly. Therefore,

$$
L=\mathfrak{U} \oplus \mathfrak{B}^{\prime}, \quad \mathfrak{A}=\bigoplus_{\alpha=0}^{n} A_{\alpha} ; \quad \mathfrak{B}^{\prime}=\bigoplus_{\alpha=0}^{n} B_{\alpha} ; \quad A_{\alpha} \equiv A, \quad B_{\alpha} \equiv B
$$

If $f \in \tilde{S_{\xi}}$, then by (1.1)

$$
\left.\left(a_{\alpha}(x)+b_{\alpha}(x)\right)^{(i)}\right|_{x=1}=\left.\left(\frac{\Delta_{\alpha}}{\Delta_{\alpha+1}}\right)^{i} \cdot\left(a_{\alpha+1}(x)+b_{\alpha+1}(x)\right)^{(i)}\right|_{x=0},
$$

and by the definition of $B$ in (2.1)

$$
a_{\alpha}^{(i)}(1)+b_{\alpha}^{(i)}(1)=\left(\frac{\Delta_{\alpha}}{\Delta_{\alpha+1}}\right)^{i} a_{\alpha+1}^{(i)}(0), \quad 0 \leqslant i<m, 0 \leqslant \alpha<n .
$$

These conditions, in terms of canonical bases (2.2) and (2.7) in $A_{\alpha}$ and $B_{\alpha}$, the same for any $\alpha, 0 \leqslant \alpha \leqslant n$, mean that

$$
S \tilde{a}_{\alpha}+\tilde{b}_{\alpha}=N_{\alpha} \tilde{a}_{\alpha+1}, \quad 0 \leqslant \alpha<n,
$$

where $S$ is the matrix (2.4) (or the mapping (2.5)) and $N_{\alpha}$ is the diagonal matrix $\left(\nu^{i} \delta_{i j}\right)_{0}^{m-1}, \nu=\nu_{\alpha}=\mu_{\alpha}^{-1}=\Delta_{\alpha} / \Delta_{\alpha+1}, 0 \leqslant \alpha<n$. We can rewrite (2.12) as

$$
\tilde{b}_{\alpha}=-S \tilde{a}_{\alpha}+N_{\alpha} \tilde{a}_{\alpha+1}, \quad 0 \leqslant \alpha<n .
$$

The coordinate $b_{n}=\operatorname{Pr}_{B} f_{n}$ in $\tilde{S}_{\xi}$ does not have any contact restriction and can be chosen in an arbitrary way. More precisely, we can forget this component of $f \in \tilde{S}_{\xi}$ because the projector on $B_{n}$ is $\left(\operatorname{Pr}_{B}\right)_{n} \cdot \tilde{P}_{k}^{n}$ and it is of local character, so its norm in $L_{\infty}$ is not bigger than $C_{2}(k, m)=\left\|\operatorname{Pr}_{B}\right\|_{\infty} \cdot C_{1}(k)$ and this estimate does not depend on $\xi$. So instead of the entire $\tilde{S}_{\xi}$ in $L$ (see (2.10)) we are interested in $\Sigma_{\xi}$ as a subspace of $L^{\prime}=\mathfrak{A} \oplus \mathfrak{B}, \mathfrak{A}=\oplus_{\alpha=0}^{n} A_{\alpha}, \mathfrak{B}=\oplus_{\alpha=0}^{n-1} B_{\alpha}$. The contact conditions (2.13) can be interpreted as the fact that $\Sigma_{\xi}$ coincides with the graph of the operator

(2.14) $\quad l: \mathfrak{A} \rightarrow \mathfrak{B}, \quad l:\left(a_{\alpha}\right)_{0}^{n} \rightarrow\left(b_{\alpha}\right)_{0}^{n-1}, \quad \tilde{b}_{\alpha}=-S a_{\alpha}+N_{\alpha} a_{\alpha+1}, \quad 0 \leqslant \alpha<n$. 
And for the estimate of the norm $\left\|q^{\prime}\right\|_{\infty}$, see (1.6), we have to analyze the orthogonal projector

$$
q: L^{\prime}=l_{n+1}^{2}(A) \oplus l_{n}^{2}(B) \rightarrow \Sigma_{\xi}=\operatorname{graph} l=\{(a, l a): a \in \mathfrak{A}\} .
$$

Recall that the inner products

$$
\left(a, a^{\prime}\right)_{\mathfrak{A}}=\sum_{0}^{n} \Delta_{\alpha} \cdot \int_{0}^{1} a_{\alpha}(x) \cdot \overline{a_{\alpha}^{\prime}(x)} d x=\sum_{0}^{n} \Delta_{\alpha} \cdot\left\langle T \tilde{a}_{\alpha}, \tilde{a}_{\alpha}^{\prime}\right\rangle
$$

and

$$
\left(b, b^{\prime}\right)_{\mathfrak{B}}=\sum_{0}^{n-1} \Delta_{\gamma} \cdot \int_{0}^{1} b_{\gamma}(x) \cdot \overline{b_{\gamma}^{\prime}(x)} d x=\sum_{0}^{n-1} \Delta_{\gamma} \cdot\left\langle V \tilde{b_{\gamma}}, \tilde{b_{\gamma}^{\prime}}\right\rangle
$$

depend heavily on $\xi$, or $\Delta$, so the notions of "orthogonality" and "adjoint operator" do depend on $\xi$.

3. Explicit Form of the Projector $q$. The projection $q$ : $\mathfrak{A} \oplus \mathfrak{B} \rightarrow \Sigma_{\xi}$ can be given explicitly in terms of the operator $l$. First of all let us recall that

$$
G^{\perp}=(\operatorname{graph} l)^{\perp}=\operatorname{graph}\left(-l^{*}\right),
$$

i.e. $(\{u, v\} ;\{x, y\})=(u, x)_{\mathfrak{A}}+(v, y)_{\mathfrak{B}}=0$ for any $\{u, v\} \in G$ iff $x=-l^{*} y$, where $l^{*}: \mathfrak{B} \rightarrow \mathfrak{A}$ and “*” is determined by (2.15) and (2.16).

Therefore, if

$$
\{a ; b\}=\{x ; l x\}+\left\{-l^{*} y, y\right\}
$$

is the (only) orthogonal decomposition of the element $f=\{a, b\} \in L^{\prime}$, with respect to the splitting $L^{\prime}=G \oplus G^{\perp} ; G \equiv \Sigma_{\xi}$, then

$$
x-l^{*} y=a ; \quad l x+y=b
$$

and

$$
\begin{aligned}
& y=\left(1+l^{*} l\right)^{-1} a+\left(1+l^{*} l\right)^{-1} l^{*} b, \\
& y=-\left(1+l l^{*}\right)^{-1} l a+\left(1+l l^{*}\right)^{-1} b
\end{aligned}
$$

or

$$
\begin{aligned}
& x=\left(1-l^{*}\left(1+l l^{*}\right)^{-1} l\right) a+l^{*}\left(1+l l^{*}\right)^{-1} b \\
& l x=+\left(1+l l^{*}\right)^{-1} l a+\left(1-\left(1+l l^{*}\right)^{-1}\right) b .
\end{aligned}
$$

Hence, the projection $q:\{a, b\} \rightarrow\{x, l x\}$ has the block representation

$$
\begin{gathered}
{\left[\begin{array}{cc}
\left(1+l^{*} l\right)^{-1} & \left(1+l^{*} l\right)^{-1} l^{*} \\
l\left(1+l^{*} l\right)^{-1} & l\left(1+l^{*} l\right)^{-1} l^{*}
\end{array}\right]} \\
=\left[\begin{array}{cc}
1-l^{*}\left(1+l l^{*}\right)^{-1} l & l^{*}\left(1+l l^{*}\right)^{-1} \\
\left(1+l l^{*}\right)^{-1} l & 1-\left(1+l l^{*}\right)^{-1}
\end{array}\right]
\end{gathered}
$$

with respect to the decomposition $\mathfrak{A} \oplus \mathfrak{B}$. The last one $\left(1=\operatorname{Pr}_{\mathfrak{A}} \oplus \operatorname{Pr}_{\mathfrak{B}}\right)$ is orthogonal for each coordinate $\alpha$ (see (2.10) and (2.1)) so $\left\|\operatorname{Pr}_{\mathfrak{A}}\right\|_{\infty},\left\|\operatorname{Pr}_{\mathfrak{B}}\right\|_{\infty} \leqslant c_{3}(k, m)$, and 
this constant $c_{3}$ does not depend on $\xi$. In particular, it means that

$$
\|q\|_{\infty} \stackrel{C_{4}(k, m)}{\sim} \quad \begin{aligned}
& \text { max of } l_{\infty} \text {-norms of four operators which are } \\
& \text { entries of the block-matrix (3.1a), }
\end{aligned}
$$

or

(3.2b) $\|q\|_{\infty} \stackrel{C_{4}}{\sim} \max$ of $l_{\infty}$-norms of four operators which are entries of the block-matrix (3.1b).

$(a \stackrel{c}{\sim} b$ means as usual that $1 / c \cdot a \leqslant b \leqslant c \cdot a$.) The second representation is more preferable because $\operatorname{dim} \mathfrak{B}<\operatorname{dim} \mathfrak{A}$ and the operator $l^{*}: \mathfrak{B} \rightarrow \mathfrak{B}$ is invertible itself $(l * l$ is not invertible).

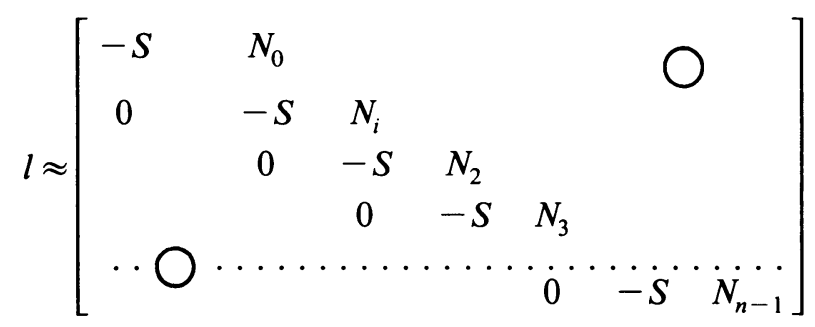

in particular, if $n=3$

$$
l \approx\left[\begin{array}{cccc}
-S & N_{0} & 0 & 0 \\
0 & -S & N_{1} & 0 \\
0 & 0 & -S & N_{2}
\end{array}\right] .
$$

The entry $l_{\gamma}^{\alpha}$ in the $\gamma$ th row and the $\alpha$ th column acting from $A_{\alpha}$ to $B_{\gamma}, \alpha=\gamma$ or $\gamma+1$.

To avoid misunderstanding recall that by the definition (2.5) $S$ acts in $A$; however, because of the special choice (2.7) of the basis $\left(v_{i}\right)_{0}^{m-1}$ in $B$ we can redefine this operator as acting from $A$ to $B$ analogously to (2.5)

$$
S: A \rightarrow B, \quad S: a \rightarrow b, \quad \tilde{b}_{j}=\sum_{0}^{k-m-1} s_{j i} a_{i}, \quad b=\sum_{0}^{m-1} \tilde{b}_{j} v_{j} .
$$

The operator (2.5) gives the extension of $a$ to the right side with the contact conditions, and by (2.7) and (2.3) the operator (3.4) gives such an element $b \in B$ that it has the same restriction at the point "l" as $a$, till the order $<m$. We use the same notation $S$ for both these operators; their matrices $m \times(k-m)$ are the same under our choice of the canonical bases (2.2) and (2.7) in $A$ and $B$.

By a standard argument $l^{*}=\left(m_{\alpha}^{\gamma}\right), m_{\alpha}^{\gamma}=\left(l_{\gamma}^{\alpha}\right)^{*}$; however, by (2.15), (2.16) we have nonstandard inner-products in these components $A_{\alpha}$ and $B_{\gamma}$ :

$$
\left(a, a^{\prime}\right)_{\alpha}=\left(a, a^{\prime}\right)_{A_{\alpha}}=\Delta_{\alpha} \cdot\left\langle T \tilde{a}, \tilde{a}^{\prime}\right\rangle,
$$

where $\tilde{a}(i)=a^{(i)}(0), 0 \leqslant i<k$, and

$$
\left(b, b^{\prime}\right)_{\gamma}=\left(b, b^{\prime}\right)_{B_{\gamma}}=\Delta_{\gamma} \cdot\left\langle V \tilde{b}, \tilde{b}^{\prime}\right\rangle,
$$

where $\tilde{b}(j)=b^{(j)}(1), 0 \leqslant j<m$. 
Therefore, if $d: A_{\alpha} \rightarrow B_{\gamma}, a \in A_{\alpha}, b \in B_{\gamma}$, then

$$
(d a, b)_{\gamma}=\Delta_{\gamma}\langle V d \tilde{a}, \tilde{b}\rangle=\Delta_{\gamma} \cdot\left\langle\tilde{a}, d^{t} V \tilde{b}\right\rangle
$$

or

and

$$
=\left(a, d^{*} b\right)_{\alpha}=\Delta_{\alpha}\left\langle T \tilde{a}, d^{*} \tilde{b}\right\rangle=\Delta_{\alpha}\left\langle\tilde{a}, T d^{*} \tilde{b}\right\rangle
$$

$$
\Delta_{\gamma} \cdot d^{t} V \tilde{b}=\Delta_{\alpha} \cdot T d^{*} \tilde{b}, \quad \forall \tilde{b}, \forall \tilde{b} \in B_{\gamma}
$$

so

$$
d^{*}=\frac{\Delta_{\gamma}}{\Delta_{\alpha}} T^{-1} d^{t} V
$$

where $d^{t}=\left(\bar{d}_{j i}\right)$ if $d=\left(d_{i j}\right)$.

In the case of the operator $l$ we are interested in pairs $(\alpha, \alpha)$ and $(\alpha, \alpha+1)$ only; in these cases

$$
\left(d_{\alpha}^{\alpha}\right)^{*}=T^{-1} d^{t} V \text { and }\left(d_{\alpha}^{\alpha+1}\right)^{*}=\nu_{\alpha} T^{-1}\left(d_{\alpha}^{\alpha+1}\right)^{t} V ; \quad \nu_{\alpha}=\frac{\Delta_{\alpha}}{\Delta_{\alpha+1}} .
$$

These remarks explain that the block-matrix representation of the operator $l^{*}$ : $\mathfrak{B}=\oplus_{0}^{n-1} B_{\gamma} \rightarrow \mathfrak{U}=\oplus_{0}^{n} A_{\alpha}$ has the form

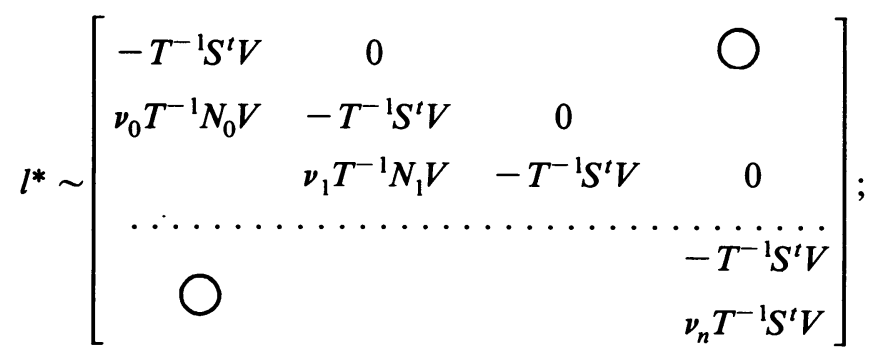

in particular, if $n=3$,

$$
l^{*} \sim\left[\begin{array}{ccc}
-T^{-1} S^{t} V & 0 & 0 \\
\nu_{0} T^{-1} N_{0} V & -T^{-1} S^{t} V & 0 \\
0 & \nu_{1} T^{-1} N_{1} V & -T^{-1} S^{t} V \\
0 & 0 & \nu_{2} T^{-1} N_{2} V
\end{array}\right] .
$$

By the statement (3.2a,b) we need to give the estimates of $l_{\infty}$-norms of the operator $H_{1}=\left(1+l^{*} l\right)^{-1}$ and its three colleagues in (3.1a) or $l_{\infty}$-norms of the operator $H=\left(1+l l^{*}\right)^{-1}$ and the other three entries in (3.1b). By (3.3) and (3.5) the block-matrix of the operator $C=1+l l^{*}: \mathfrak{B} \rightarrow \mathfrak{B}$ has the form $(n=3)$

$$
1+l l^{*} \approx\left[\begin{array}{ccc}
1+S T^{-1} S^{t} V & -N_{0} T^{-1} S^{t} V & \bigcirc \\
+\nu_{0} N_{0} T^{-1} N_{0} V & & \\
-\nu_{0} S T^{-1} N_{0} V & 1+S T^{-1} S^{t} V & -N_{1} T^{-1} S^{t} V \\
& +\nu_{1} N_{1} T^{-1} N_{1} V & \\
& -\nu_{1} S T^{-1} N_{1} V & 1+S T^{-1} S^{t} V \\
& & +\nu_{2} N_{2} T^{-1} N_{2} V
\end{array}\right]
$$


and for any $n$

$$
\left\{\begin{array}{l}
C=\left(C_{\gamma}^{\alpha}\right)_{0}^{n-1}, \quad C_{\gamma}^{\alpha}=0, \quad|\alpha-\gamma|>1, \\
C_{\alpha}^{\alpha}=1+S T^{-1} S^{t} V+\nu_{\alpha} N_{\alpha} T^{-1} N_{\alpha} V, \quad 0 \leqslant \alpha<n, \\
C_{\alpha}^{\alpha+1}=-N_{\alpha} T^{-1} S^{t} V, \quad C_{\alpha}^{\alpha-1}=-\nu_{\alpha-1} S T^{-1} N_{\alpha-1} V .
\end{array}\right.
$$

Of course, the operator $H=C^{-1}=\left(1+l l^{*}\right)^{-1}$ is of norm $\leqslant 1$ for any mesh $\xi$ as an operator in the Hilbert space $\mathscr{B}=l_{2}^{n}\left(B_{\alpha},\left\{\Delta_{\alpha}\right\}\right)$; however, we need to estimate the norm $\|H\|_{\infty}=\left\|H: l_{\infty}^{n}\left(B_{\alpha}\right) \rightarrow l_{\infty}^{n}\left(B_{\alpha}\right)\right\|$. Recall that this norm $\|H\|_{\infty}=$ $\max _{\alpha} \sum_{\gamma=0}^{n-1}\left|H_{\alpha}^{\gamma}\right|$, where $|h|, h: B_{\gamma} \rightarrow B_{\alpha}$, is a norm of an operator (matrix) in $m$-dimensional Hilbert space. It could be useful to consider $H^{t}$ as an operator in $l_{1}^{n}\left(B_{\alpha}\right)$; then $\left(H^{t}\right)_{\gamma}^{\alpha}=\left(H_{\alpha}^{\gamma}\right)^{t}$ and $\left\|H^{t}\right\|_{1}=\|H\|_{\infty}$.

4. Blocks of $q$ and Their Invertibility. Now we would like to use all the advantages of the representation (3.6)-(3.7) to get the estimates of $\|H\|_{\infty},\|H l\|_{\infty},\left\|l^{*} H\right\|_{\infty}$ and $\left\|l^{*} H l\right\|_{\infty}$. First of all, instead of $H$ itself we will analyze $\tilde{V} \cdot H$, where $\tilde{D}:\left(b_{\alpha}\right)_{0}^{n-1} \rightarrow$ $\left(D b_{\alpha}\right)_{0}^{n-1}$ denotes the block-diagonal operator for any $D: B \rightarrow B$. So we are interested in the block-matrix $\check{C}=C \cdot \widetilde{V^{-1}}(n=3)$

$$
\check{C}=\left[\begin{array}{ccc}
V^{-1}+S T^{-1} S^{t} & -N_{0} T^{-1} S t & 0 \\
+\nu_{0} N_{0} T^{-1} N_{0} & & \\
-\nu_{0} S T^{-1} N_{0} & V^{-1}+S T^{-1} S^{t} & -N_{1} T^{-1} S^{t} \\
& +\nu_{1} N_{1} T^{-1} N_{1} & \\
0 & -\nu_{1} S T^{-1} N_{1} & V^{-1}+S T^{-1} S^{t} \\
& & +\nu_{2} N_{2} T^{-1} N_{2}
\end{array}\right] .
$$

The general case (3.7) needs the appropriate adjustment. So

$$
\begin{gathered}
H=C^{-1}=(\check{C} \tilde{V})^{-1}=\tilde{V}^{-1} \cdot \check{C}^{-1} \\
l^{*} H=\left(\tilde{T}^{-1}\left(\nu \tilde{N}-\tilde{S}^{t}\right) \tilde{V}\right)\left(\tilde{V}^{-1} \cdot \check{C}^{-1}\right)=\tilde{T}^{-1} \cdot\left(\nu \tilde{N}-\tilde{S}^{t}\right) \check{C}^{-1}
\end{gathered}
$$

where

$$
\widetilde{\nu N}=\left[\begin{array}{cccc}
0 & 0 & \cdots & 0 \\
\nu_{0} N_{0} & & & \bigcirc \\
& \nu_{1} N_{1} & & \\
\bigcirc & & & \cdot \nu_{n-1} N_{n-1}
\end{array}\right]
$$

and if the matrix does not depend on $\boldsymbol{\xi}$ the symbol $\tilde{\boldsymbol{P}}$ means the corresponding diagonal matrix ( $n$ by $n$, or $n \times(n+1)$, or $(n+1) \times n$ ) with entries $P$ on the diagonal.

$$
\begin{gathered}
H l=\tilde{V}^{-1} \cdot \check{C}^{-1}(-\tilde{S}+\tilde{N}), \text { where }(\text { compare }(3.3)) \\
\tilde{N}=\left[\begin{array}{cccccc}
0 & N_{0} & & & & \\
& 0 & N_{1} & & & \bigcirc \\
\cdot & \cdot & \cdot & \cdot & \cdot & \cdot \\
0 & & & 0 & N_{n-1}
\end{array}\right]
\end{gathered}
$$


and $l^{*} H l$ can be decomposed by the same way:

$$
l^{*}=\tilde{T}^{-1}\left(\nu \tilde{N}-\tilde{S}^{t}\right) \check{C}^{-1}(-\tilde{S}+\tilde{N}) .
$$

Many terms of these operators (4.2)-(4.7) do not depend on $\xi$; these factors or terms have the same norm as their generators $T^{-1}, V^{-1}$, or $S$ and $S^{t}$ in the block-space $A$ or $B$. ( $T^{-1}$ and $V^{-1}$ do exist as inverses of Gramians.) It means that their estimates do not depend on the mesh $\xi$ but on $k$ and $m$ only.

Therefore, if we know a good estimate of the norm $\rho=\left\|\check{C}^{-1}\right\|_{\infty}$ then we can (have to) analyze instead of the three operators

$$
l^{*}\left(1+l l^{*}\right)^{-1}, \quad\left(1+l l^{*}\right)^{-1} l, \quad l^{*}\left(1+l l^{*}\right)^{-1} l
$$

only their "parts" (see (4.3), (4.5), (4.7))

$$
\nu \tilde{N} \cdot \check{C}^{-1}, \quad \check{C}^{-1} \cdot \tilde{N}, \quad \nu \tilde{N} \cdot C^{-1} \cdot \tilde{N}
$$

Now we can formulate the main problem. Let $T, V$ and $S$ be matrices (2.6), (2.8) and (2.4), correspondingly. For any mesh (0.1) they produce the block-matrix $\check{C}$ by (4.1) and (3.7) and we consider it as an operator in $l_{n}^{\infty}\left(\mathbf{C}^{n}\right) \rightarrow l_{n}^{\infty}\left(\mathbf{C}^{m}\right)$.

Problem. Find good estimates for the norm $\rho=\left\|\check{C}^{-1}\right\|_{\infty}$ and norms of its relatives (4.8). Does there exist such a constant $C(k, m)$ that these norms do not exceed it for any choice $\nu=\left(\nu_{0}, \nu_{1}, \ldots, \nu_{n-1}\right)$ ?

In this question $\nu_{i}$ could be "zero" too. It is not a more general question than the initial one. Indeed if these operators are uniformly bounded for any $\nu, 0<\nu_{i}<\infty$, $0 \leqslant i<n$, then by the compactness argument, for any fixed $n$, the operator $\check{C}$ has to be invertible if some $\nu_{i}=0$ and $\left\|\check{C}^{-1}\right\|_{\infty}$ has the same bound.

For further explicit calculation we need to know the matrices $V^{-1}+S T^{-1} S^{t}$ and $S T^{-1}$. We give now the results of this computation in the cases $k=2,4,6 ; m=k / 2$. Recall (2.9.1)-(2.9.3) in Section 2.

(4.9.1) $k=2 ; \quad V^{-1}=3 ; \quad T^{-1}=4, \quad V^{-1}+S T^{-1} S^{t}=4 ; \quad S T^{-1}=-2$.

(4.9.2) $\quad k=4 ; \operatorname{det} V=1 / 1260 ; \quad \operatorname{det} T=1 / 4800$,

$$
\begin{gathered}
V^{-1}=\left[\begin{array}{rr}
12 & 66 \\
66 & 468
\end{array}\right], \quad T^{-1}=\left[\begin{array}{rr}
16 & -120 \\
-120 & 1200
\end{array}\right], \\
S T^{-1}=\left[\begin{array}{rr}
-4 & 60 \\
-60 & 840
\end{array}\right], \quad V^{-1}+S T^{-1} S^{t}=\left[\begin{array}{rr}
16 & 120 \\
120 & 1200
\end{array}\right] .
\end{gathered}
$$

(4.9.3) $\quad k=6 ; \quad \operatorname{det} V=474320 \cdot(55440)^{-3} ; \quad \operatorname{det} T=(62720 \cdot 27 \cdot 4900)^{-1}$,

$$
\begin{gathered}
V^{-1}=\left[\begin{array}{rrr}
27 & 351 & 2722 \\
351 & 6003 & 52596 \\
2772 & 52596 & 484272
\end{array}\right], \quad T^{-1}=\left[\begin{array}{rrr}
36 & -630 & 6720 \\
-630 & 14700 & -176400 \\
6720 & -176400 & 2257920
\end{array}\right], \\
-S T^{-1}=\left[\begin{array}{rrr}
6 & -210 & 3360 \\
210 & -7140 & -110880 \\
3360 & -110880 & 1673280
\end{array}\right], \\
S T^{-1} S^{t}=\left[\begin{array}{rrr}
9 & 279 & 3948 \\
279 & 8697 & 123804 \\
3948 & 123804 & 1773648
\end{array}\right] \\
V^{-1}+S T^{-1} S^{t}=\left[\begin{array}{rrr}
36 & 630 & 6720 \\
630 & 14700 & 176400 \\
6720 & 176400 & 2257920
\end{array}\right]
\end{gathered}
$$


Remark. Put $J: \mathbf{R}^{m} \rightarrow \mathbf{R}^{m}, J:\left(x_{i}\right)_{0}^{m-1} \rightarrow\left((-1)^{i} x_{i}\right)_{0}^{m-1}$. Our numerical data show that

$$
V^{-1}+S T^{-1} S^{t}=J T^{-1} J
$$

and

$$
T+S^{t} V S=J V J,
$$

if $k=2 m=2,4$, or 6 . I conjecture that $(4.10 \mathrm{a}, \mathrm{b})$ hold for any $m>3, k=2 m$, also (see (5.7) below).

5. The Case of the Geometric Mesh. If the partition $\xi=\left(\xi_{0}=0<\xi_{1}<\cdots<\xi_{n}\right.$ $\left.<\xi_{n+1}=1\right)$ is a geometric mesh, i.e. $\mu_{\alpha}=\left(\xi_{\alpha+2}-\xi_{\alpha+1}\right) /\left(\xi_{\alpha+1}-\xi_{\alpha}\right) \equiv \mu$ for any $\alpha, 0 \leqslant \alpha<n$, then $\nu_{\alpha}=\nu=1 / \mu$ in (4.1) and all the operators

$$
N_{\alpha} \equiv N=\left\{\nu^{i} \delta_{i j}\right\}_{0}^{m-1}=\left(\begin{array}{llll}
1 & & & \\
& \nu & & \\
& \nu^{2} & & \\
& & \ddots & \nu^{m-1}
\end{array}\right)
$$

are the same. The matrix $\check{C}$ becomes a Toeplitz block-matrix with three diagonals, and its common row is

$$
0 \cdots 0-\nu S T^{-1} N \quad V^{-1}+S T^{-1} S^{t}+\nu N T^{-1} N \quad-N T^{-1} S^{t} 0 \cdots 0
$$

with $(m \times m)$-matrix entries.

The uniform boundedness (for a fixed $\nu$ with respect to $n$ ) of the inverses $\check{C}^{-1}$ depends heavily on the structure of singularities of the rational (or quadratic) pencil

$$
W(z)=-\nu F z+D-F^{t} \frac{1}{z}
$$

where

$$
F=S T^{-1} N, \quad D=V^{-1}+S T^{-1} S^{t}+\nu N T^{-1} N .
$$

For a detailed analysis see I. Gohberg and I. Feldman [8, Chapter 8]. Now we will use some general results in this connection.

LEMMA 5.1. Let

$$
V(z)=D_{1} z+D_{0}+D_{-1} \cdot \frac{1}{z}
$$

be a pencil, where $D_{j},|j| \leqslant 1$, are $(m \times m)$-matrices. The following conditions are equivalent:

(a) there exist both left and right factorizations of special type

$$
V(z)=\left(N_{1} z+1\right) N_{0}\left(1+N_{-1} \frac{1}{z}\right)
$$

where $\operatorname{spectr} N_{ \pm 1} \subset D=\{|z|<1\}$ and $N_{0}$ invertible, and

$$
V(z)=\left(M_{-1} \frac{1}{z}+1\right) M_{0}\left(M_{1} z+1\right),
$$

where $\operatorname{spectr} M_{ \pm 1} \subset D$ and $M_{0}$ nonsingular; 
(b) for $n$ sufficiently large the system

$$
\begin{aligned}
& D_{0} x_{0}+D_{-1} x_{1}=y_{0}, \\
& D_{1} x_{0}+D_{0} x_{1}+D_{-1} x_{2}=y_{1}, \\
& \ldots \ldots \ldots \ldots \ldots \\
& D_{1} x_{n-2}+D_{0} x_{n-1}+D_{-1} x_{n}=y_{n-1}, \\
& D_{1} x_{n-1}+D_{0} x_{n}=y_{n},
\end{aligned}
$$

has a solution $x=\left(x_{i}\right)_{0}^{n}, x_{i} \in \mathbf{C}^{m}, 0 \leqslant i \leqslant n$ for any $y=\left(y_{i}\right)_{0}^{n}, y_{i} \in C^{m}, 0 \leqslant i \leqslant n$, and there exists a constant $R$ such that

$$
\|x\|_{\infty}=\sup _{0 \leqslant i<n}\left|x_{i}\right| \leqslant R \cdot\|y\|_{\infty} \text { for any } n \geqslant n_{0},
$$

i.e. $R$ does not depend on $n \geqslant n_{0}$.

(c) The same is true for the $l^{2}$-norm also, i.e. the inequality

$$
\sum_{0}^{n}\left|x_{i}\right|^{2} \leqslant R^{2} \sum_{0}\left|y_{i}\right|^{2}, \quad n \geqslant n_{0},
$$

holds for the solution of (5.1.b). (The constant $R$ in (5.1.b.2) could be different than in

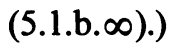

We can transform these inequalities to the case of the weight spaces $l^{2}$ or $l^{\infty}$. If $\mu>0$ and $y_{i}=y_{i}^{\prime} \mu^{i / 2}, x_{i}=x_{i}^{\prime} \mu^{i / 2}, 0 \leqslant i \leqslant n$, then the system (5.1.b) becomes

$$
D_{0}^{\prime} x_{0}^{\prime}+D_{-1}^{\prime} x_{1}^{\prime}=y_{0}^{\prime} \text {, }
$$

$$
\begin{aligned}
& D_{1}^{\prime} x_{i-1}^{\prime}+D_{0}^{\prime} x_{i}^{\prime}+D_{-1}^{\prime} x_{i+1}=y_{i}^{\prime}, \quad 0<i<n, \\
& \cdots \cdots \cdots \cdots \cdots \cdots \cdots \\
& D_{1}^{\prime} x_{n-1}^{\prime}+D_{0}^{\prime} x_{n}^{\prime}=y_{n}^{\prime}
\end{aligned}
$$

where

$$
D_{1}^{\prime}=D_{1} \mu^{-1 / 2}, \quad D_{0}^{\prime}=D_{0}, \quad D_{-1}^{\prime}=D_{-1} \mu^{1 / 2} .
$$

Therefore, the conditions of Lemma 5.1 are equivalent to the following condition

(5.1.d) for $n \geqslant n_{0}, n_{0}$ sufficiently large, for any $\mu>0$ the system (5.1.b') has a (unique) solution $x^{\prime}=\left(x_{i}^{\prime}\right)_{0}^{n}, x_{i}^{\prime} \in \mathbf{C}^{m}, 0 \leqslant i \leqslant n$, and there exists a constant $R$ such that

$$
\sup _{0<i \leqslant n}\left|x_{i}^{\prime}\right| \mu^{i / 2} \leqslant R \cdot \sup _{0 \leqslant i \leqslant n}\left|y_{i}^{\prime}\right| \mu^{i / 2}
$$

and

$$
\sum_{0}^{n}\left|x_{i}^{\prime}\right|^{2} \mu^{i} \leqslant R^{2} \cdot \sum_{0}^{n}\left|y_{i}^{\prime}\right|^{2} \mu^{i},
$$

$R$ does not depend on $n$ (or on $\mu$ ).

Hence, (5.1.d) implies that the pencil $D(z)=D_{1} z+D_{0}+D_{-1} 1 / z$ has both the factorizations of type (5.1.a.l) and (5.1.a.r), and by (5.1.b") it means that $\left(\zeta=\mu^{1 / 2} z\right)$ the pencil $D^{\prime}(\zeta)=D_{i}^{\prime} \zeta+D_{0}^{\prime}+D_{-1}^{\prime} 1 / \zeta$ has the factorizations of the same type with conditions

$$
\begin{array}{ll}
\operatorname{spectr} N_{-1}^{\prime}, & \operatorname{spectr} M_{-1}^{\prime} \subset D_{\sqrt{\mu}}=\left\{z:|z|<\mu^{1 / 2}\right\} \\
\operatorname{spectr} N_{+1}^{\prime}, & \operatorname{spectr} M_{+1}^{\prime} \subset D_{1 / \sqrt{\mu}}=\left\{z:|z|<\mu^{-1 / 2}\right\}
\end{array}
$$


This form (5.1.d) of Lemma 5.1 will be used to realize why the condition (5.3.b) below is too important (and maybe the only concrete fact that we do need).

LEMMA 5.2. The pencil (5.1.5) has both the factorizations (5.1.a.l) and (5.1.a.r) if

$$
\operatorname{Re}\langle V(z) \omega, \omega\rangle<0, \quad \forall z,|z|=1 ; \omega \in \mathbf{C}^{m}, \omega \neq 0 \text {. }
$$

These results on the discrete multi-dimensional Hopf-Wiener equation (see [8, Chapter 8]) help us right now. In [10] I give independent analysis of the pencil that appears in our problem on the piecewise-polynomial approximant and its generalization; there I get some new results on factorization of rational pencils that can be useful for other applications.

LEMMA 5.3. The pencil (5.1), if $\nu=1$, has the property (5.2.a). Indeed, in this case

$$
\begin{aligned}
\left\langle\left(-S T^{-1} z\right.\right. & \left.\left.+V^{-1}+S T^{-1} S^{t}+T^{-1}-T^{-1} S^{t} \bar{z}\right) \omega, \omega\right\rangle \\
& =\left\langle V^{-1} \omega, \omega\right\rangle+\left\langle T^{-1} S^{t} \omega, \omega\right\rangle+\left\langle T^{-1} \omega, \omega\right\rangle-2 \operatorname{Re} z\left\langle T^{-1} \omega, S^{t} \omega\right\rangle .
\end{aligned}
$$

Both $V^{-1}$ and $T^{-1}$ are strictly-positive definite, so $\left\langle V^{-1} \omega, \omega\right\rangle>0$, and by the Cauchy inequality for the inner product,

$$
[u, v]=\left\langle T^{-1} u, v\right\rangle \quad\left|2 \operatorname{Re} z\left\langle T^{-1} \omega, S^{t} \omega\right\rangle\right| \leqslant\left\langle T^{-1} \omega, \omega\right\rangle+\left\langle T^{-1} S^{t} \omega\right\rangle
$$

and the lemma holds.

In the case of the geometric mesh we can be interested in $\nu \in(0,1]$ instead of $\nu \in(0, \infty)$ because the original problems for $\nu$ and $1 / \nu \cdot($ or $\mu$ and $1 / \mu$ ) are equivalent; this change means only that we run over $[0,1]$ from 1 to 0 . And if $\nu \in(0,1]$, the factors $\tilde{N}$ and $\nu \widetilde{N}$ as operators in $l_{n}^{\infty}\left(C^{k-m}\right)$ have norms 1 so we only have to consider (see (4.8)) $\check{C}$ and $\check{C}^{-1}$. The operators depend on the parameter $\nu \in[0,1]$; by Lemmas 5.1-5.3 it is nice if $\nu=1$ (the uniform mesh).

We could complete the analysis of the geometric mesh if we knew that

$$
\check{C}(0) \text { is invertible for any } n \text { and }\left\|\check{C}^{-1}(0)\right\|_{\infty} \leqslant R(0)<\infty
$$
independently on $n$,

$$
\begin{aligned}
& w_{\nu}(z)=\operatorname{det} W(z), W \text { as in (5.1), does not vanish on }\{z:|z|=1\} \\
& \text { for any } \nu, 0<\nu \leqslant 1 .
\end{aligned}
$$

Indeed, if $\nu$ is small enough (see (4.1)), we can consider $\check{C}$ as a perturbation of $\check{C}(0)$; more precisely, if $\nu \leqslant \frac{1}{2}\left(\delta(0) \cdot\left(\left\|T^{-1}\right\|+2\left\|T^{-1} S^{t}\right\|\right)\right)^{-1}=\nu_{*}$, then by the Neumann-series $\check{C}$ is invertible and $\left\|\check{C}^{-1}\right\|_{\infty} \leqslant 2 \cdot R(0)$.

For $\nu \in\left[\nu_{*}, 1\right]$ we will use essentially the fact that the pencil $(5.1)$ is generated by the orthogonal projection in $l_{2}\left(P_{j} ; \Delta\right)=\left\{f=\left(f_{\alpha}\right)_{0}^{n+1}: \Sigma_{0}^{n+1}\left\|f_{\alpha}\right\|^{2} \mu^{\alpha}=\|f\|^{2}\right\}, \mu \cdot \nu$ $=1$. This projection is certainly bounded and its norm is 1 . It implies that all the operators in (3.1a), (3.1b) and so on are uniformly bounded with respect to $n$ or $\mu$ if we consider them in the Hilbert weight spaces $\Sigma_{n+1}\left(\mathbf{C}^{m} ; \Delta(\mu)\right)$. In particular it means that the system (5.1.d) for $D_{1}^{\prime}=-\nu F, D_{0}^{\prime}=D, D_{-1}^{\prime}=-F^{t}$, - see (5.1) for the further concretization of these matrices, - are uniformly solvable, $n \geqslant n_{0}(\mu)$, in $l_{n+1}\left(\mathbf{C}^{m} ; \Delta(\mu)\right)$, i.e. (5.1.d) holds. 
Therefore, by Lemma 5.1.d and the subsequent remarks there exist two factorizations

$$
V(z)=\left(N_{1}^{\prime} z+1\right) N_{0}^{\prime}\left(1+N_{-1}^{\prime} \frac{1}{z}\right)=\left(1+M_{-1}^{\prime} \frac{1}{z}\right) M_{0}^{\prime}\left(M_{1}^{\prime} z+1\right)
$$

with conditions (5.1.d $)$ where $N_{0}^{\prime}, M_{0}^{\prime}$ are nonsingular $(m \times m)$-matrices.

At the initial moment $\nu=1$ the determinant $w_{1}(z)$ by Lemma 5.3 has $m$ zeros inside the disc $\mathscr{D}_{1}$ and $m$ zeros outside it. When $\nu$ changes the coefficient of $z^{m}$ is equal to $(-1)^{m} \nu^{m}$ det $F$ and is not zero (all the factors $S, T^{-1}$ and $N$ of $F$ are nonsingular matrices), so the zeros of $w_{\nu}(z)$ depend continuously on the parameter $\nu$ and by the condition (5.3.b) $m$ of them will stay inside $\mathscr{D}_{1}$ and $m$ others will be outside $\mathscr{D}_{1}$ for any $\nu, 0<\nu \leqslant 1$. By (5.3.c) we do know that $m$ singular points of $V$ are outside the disk $\mathscr{D}_{\sqrt{\mu}}, \mu=1 / \nu>1$, so we can be sure that $V_{\nu}$ has no singular points in the annulus $\left\{z: 1 \leqslant|z| \leqslant \mu^{1 / 2}\right\}$; indeed, their number is $2 m$ and $m$ of them belong to $\mathscr{D}_{1}$ and $m$ others are outside $\overline{\mathscr{D}}_{\sqrt{\mu}}$. Therefore, the factors $\left(1+N_{-1}^{\prime} 1 / z\right)$ and $\left(1+M_{-1}^{\prime} 1 / z\right)$ are invertible not only for $|z| \geqslant \sqrt{\mu}$ but for any $z,|z| \geqslant 1$; indeed, by (5.3.c) $\left(1+N_{-1}^{\prime} 1 / z\right)^{-1}=V(z)^{-1}\left(N_{1}^{\prime} z+1\right) N_{0}^{\prime}$ for $1 \leqslant|z| \leqslant \sqrt{\mu}$ because det $V_{\nu}(z) \neq$ 0 in this annulus. (The analogous formula could be written for the factor of the second factorization.) It shows that the first line of $\left(5.1 . \mathrm{d}^{\prime}\right)$ could be changed to the stronger form

$$
\operatorname{spectr} N_{-}^{\prime}, \quad \operatorname{spectr} M_{-}^{\prime} \subset \mathscr{D}_{1}=\{z:|z|<1\}
$$

and together with the second line $\left(\nu<1\right.$ so $\left.\mu>1, \mu^{-1 / 2}=\nu^{1 / 2}<1\right)$ it means that the spectra of all four matrices $N_{ \pm 1}, M_{ \pm 1}$ satisfy the conditions (a) of Lemma 5.1. Hence, by this lemma the condition (b) holds also for $D_{1}=\nu S T^{-1} N, D_{-1}=$ $-N T^{-1} S^{t}, D_{0}=V^{-1}+S T^{-1} S^{t}+\nu N T^{-1} N$ and it is precisely what we do need. So all the operators $\check{C}, \nu_{*} \leqslant \nu \leqslant 1$, are invertible, $\left\|C^{-1}\right\|_{\infty} \leqslant R(\nu), n \geqslant n_{*}$, and the Neumann-series argument shows that for the closed interval $\left[\nu_{*}, 1\right]$ the upper bound $R_{*} \geqslant R(\nu), \nu_{*} \leqslant \nu \leqslant 1$, could be chosen as finite and independent on $\nu$ and $n$. Now $C(k)=\max \left\{2 R(0) ; R_{*}\right\}$ gives the bound of $L_{\infty}$-norms of orthogonal projectors in the case of the geometric mesh.

However this conclusion depends heavily on the assumptions (5.3.a) and (5.3.b). And now we check these conditions in the case $m=1,2,3 ; k=2 m$, by explicit analysis of our concrete matrices.

If $\nu=0$, the matrix $\check{C}$ in (4.1) loses its lower diagonal, the entry on the main diagonal is $D_{0}=V^{-1}+S T^{-1} S^{t}$, and on the upper diagonal the entry matrix

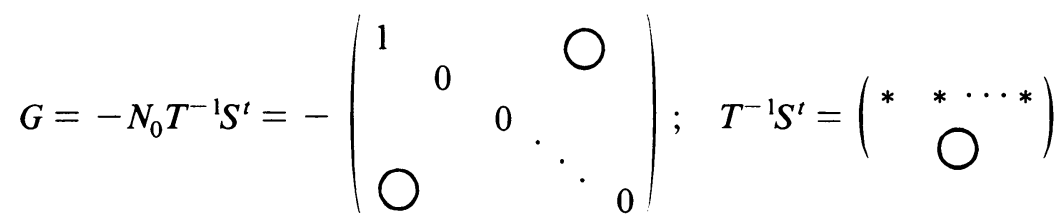

has one nonzero row only. $D_{0}$ does not depend on $\nu$ or $n$, so

$$
\check{C}(0)=\left[\begin{array}{cccccc}
D_{0} & G & & & \\
0 & D_{0} & G & & \\
0 & 0 & D_{0} & G & \\
\cdots & \cdots & \cdots & \cdots & \ldots \\
0 & 0 & & & D_{0}
\end{array}\right]=\left[\begin{array}{ccccc}
1 & G D_{0}^{-1} & & & \\
0 & 1 & G D_{0}^{-1} & & \\
& & & G D_{0}^{-1} & \\
& & & & 1
\end{array}\right] \cdot \tilde{D}_{0}
$$


and these operators are certainly uniformly invertible if the spectrum $\sigma\left(G D_{0}^{-1}\right)<1$. This operator $G D_{0}^{-1}$ is one-dimensional, its matrix is

$$
\left[\begin{array}{c}
\gamma_{0}, \gamma_{1}, \ldots, \gamma_{m-1} \\
\bigcirc
\end{array}\right]
$$

and the only nonzero eigenvalue is

$$
\gamma_{0}=\sum_{j=0}^{m-1}\left(T^{-1} S^{t}\right)_{0 j}\left(D_{0}^{-1}\right)_{j 0}
$$

The computations show that for $k=2 m$,

$$
\begin{aligned}
& k=2, \quad \gamma_{0}=-\frac{1}{2} \\
& k=4, \quad \gamma_{0}=-4 \cdot \frac{1}{4}+(-60) \cdot\left(-\frac{1}{40}\right)=\frac{1}{2} \\
& k=6, \quad \gamma_{0}=6 \cdot \frac{1}{4}+210 \cdot\left(-\frac{1}{35}\right)+3360 \cdot \frac{1}{672}=-\frac{1}{2} .
\end{aligned}
$$

This suggests that in the general case $\gamma_{0}=(-1)^{k / 2} \cdot \frac{1}{2}$, and it would be interesting to check this fact.

The last step is to examine the property (5.3.b). We present explicit computation of these rational functions $(k=2,4,6)$ and then it is clear that (5.3.b) holds.

$$
\begin{aligned}
& k=2 . \\
& w_{\nu}(z)=2 \nu z+4(1+\nu)+2 \cdot \frac{1}{z}
\end{aligned}
$$

and $|z|=1 \Rightarrow\left|w_{v}(z)\right| \geqslant 4-2|\bar{z}|+4 \nu-|2 \nu z|=2(1+\nu) \geqslant 2$.

$$
\begin{aligned}
& \left.\begin{array}{l}
k=4 . \\
w_{\nu}(z)=\operatorname{det}\left\{2 \nu\left(\begin{array}{cc}
4 & -60 \nu \\
60 & -840 \nu
\end{array}\right)+\left(\begin{array}{rr}
16(1+\nu) & 120\left(1-\nu^{2}\right) \\
120\left(1-\nu^{2}\right) & 1200\left(1+\nu^{3}\right)
\end{array}\right)\right. \\
+\left(\begin{array}{cc}
4 & 60 \\
-60 \nu & -840 \nu
\end{array}\right) \cdot \frac{1}{z}
\end{array}\right\} \\
& =240 \operatorname{det}\left\{\begin{array}{rr}
\nu \cdot z+4(1+\nu)+1 \cdot \frac{1}{z} & -15 \nu^{2} \cdot z+30\left(1-\nu^{2}\right)+15 \cdot \frac{1}{z} \\
\nu \cdot z+2\left(1-\nu^{2}\right)-1 \cdot \frac{1}{z} & -14 \nu^{2} \cdot z+20\left(1-\nu^{2}\right)-14 \cdot \frac{1}{z}
\end{array}\right\}
\end{aligned}
$$

and

$$
w_{\nu}(z)=240 \cdot \sum_{i=-2}^{2} p_{i}(\nu) z^{i} ; \quad p_{i}(0)=\sum_{k=0}^{4} p_{i k} \nu^{k}
$$

where $p_{i k}$ are given in Table 1 . 
TABLE 1

\begin{tabular}{rrrrrr}
\hline${ }_{i}{ }^{k}$ & \multicolumn{1}{c}{4} & \multicolumn{1}{c}{3} & \multicolumn{1}{c}{2} & \multicolumn{1}{c}{1} & \multicolumn{1}{c}{0} \\
\hline 0 & 20 & 65 & 92 & 65 & 20 \\
+1 & -10 & -26 & -26 & -10 & \\
-1 & & -10 & -26 & -26 & -10 \\
+2 & & 1 & & & \\
-2 & & & & 1 & 10 \\
$\pi$ & 10 & 28 & 40 & 28 & 10 \\
\hline
\end{tabular}

The last row $\pi_{k}=p_{0 k}-\Sigma_{0<|i|<2}\left|p_{i k}\right|$ contains strictly positive scalars only, and it implies for $|z|=1$ that

$$
\left|w_{\nu}(z)\right| \geqslant 240\left(p_{0}(\nu)-\sum_{0<|i|<2}\left|p_{i}(0)\right|\right)=240 \cdot \sum_{k=0}^{4} \pi_{k} \nu^{k} \geqslant 2400>0 .
$$

(5.5.3) $k=6$

$$
\begin{aligned}
& w_{0}(z)=\operatorname{det}\left\{+\nu z\left(\begin{array}{rrr}
6 & -210 \nu & 3360 \nu^{2} \\
210 & -7140 \nu & 11088 \nu^{2} \\
3360 & -110880 \nu & 1673280 \nu^{2}
\end{array}\right)\right. \\
& +\left(\begin{array}{rrr}
36(1+\nu) & 630\left(1-\nu^{2}\right) & 6720\left(1+\nu^{3}\right) \\
630\left(1-\nu^{2}\right) & 14700\left(1+\nu^{3}\right) & 176400\left(1-\nu^{4}\right) \\
6720\left(1+\nu^{3}\right) & 176400\left(1+\nu^{4}\right) & 2257920\left(1+\nu^{5}\right)
\end{array}\right) \\
& \left.+\frac{1}{z}\left(\begin{array}{rrr}
6 & 210 & 3360 \\
-210 \nu & -7140 \nu & -110880 \nu \\
3360 \nu^{2} & 110880 \nu^{2} & 1673280 \nu^{2}
\end{array}\right)\right\} \\
& =6 \cdot 210 \cdot 3360 \cdot\left[\begin{array}{rr}
\nu z+6(1+\nu)+\frac{1}{z} & -35 \nu^{2} z+105\left(1-\nu^{2}\right)+35 \cdot \frac{1}{z} \\
\nu z+3\left(1-\nu^{2}\right)-\nu \cdot \frac{1}{z} & -34 \nu^{2} z+70\left(1+\nu^{3}\right)-34 \nu \cdot \frac{1}{z} \\
\nu z+2\left(1+\nu^{3}\right)+\nu^{2} \cdot \frac{1}{z} & -33 \nu^{2} z+52.5\left(1-\nu^{4}\right)+33 \nu^{2} \cdot \frac{1}{z}
\end{array}\right. \\
& 560 \nu^{3} z+1120\left(1+\nu^{3}\right)+560 \cdot \frac{1}{z} \\
& 528 \nu^{3} z+840\left(1-\nu^{4}\right)-528 \nu \cdot \frac{1}{z} \\
& \left.498 \nu^{3} z+672\left(1+\nu^{5}\right)+498 v^{2} \cdot \frac{1}{z}\right] \\
& =\gamma \cdot \sum_{-3}^{3} p_{i}(\nu) \cdot z^{i}=\gamma \cdot \sum_{i=-3}^{3}\left(\sum_{k=0}^{9} p_{i k} \nu^{k}\right) z^{i},
\end{aligned}
$$

where $\gamma=2 \cdot 6 \cdot 210 \cdot 3360=8,467,200$, and the polynomials $p_{i},|i| \leqslant 3$, are given in Table 2. 
TABLE 2

Table of the Coefficients $p_{i k}$ of $\Sigma_{-3}^{3} P_{i}(x) \cdot z^{i}=\Sigma_{-3}^{3}\left(\Sigma_{0}^{9} p_{i k} x^{k}\right) z^{i}$

\begin{tabular}{crrrrrrrrrr}
\hline$i / k$ & 9 & \multicolumn{1}{c}{8} & \multicolumn{1}{c}{7} & \multicolumn{1}{c}{6} & \multicolumn{1}{c}{5} & \multicolumn{1}{c}{4} & \multicolumn{1}{c}{3} & \multicolumn{1}{c}{1} & \multicolumn{1}{c}{0} \\
\hline 0 & 980 & 6860 & 22792 & 47058 & 66426 & 66426 & 47058 & 22792 & 6860 & 980 \\
+1 & 490 & 3136 & 9401 & 17153 & 20799 & 17153 & 9401 & 3136 & 490 & \\
-1 & & 490 & 3136 & 9401 & 17153 & 20153 & 17153 & 9401 & 3136 & 490 \\
+2 & 0 & 56 & 245 & 461 & 461 & 245 & 56 & & & \\
-2 & & & & 56 & 245 & 461 & 461 & 245 & 56 & 0 \\
+3 & & & & 1 & & & & & & \\
-3 & & & & & & & 1 & & & \\
\hline$\left(\Sigma_{i} p_{i k}\right)-p_{0 k}$ & 490 & 3682 & 12782 & 27072 & 38651 & 38651 & 27072 & 12782 & 3682 & 490 \\
\hline$\frac{\Sigma_{k}-p_{0 k}}{p_{0 k}}$ & $=.5$ & .537 & .561 & .576 & .582 & .582 & .576 & .561 & .537 & $=.5$ \\
\hline
\end{tabular}

This table shows that for any $z,|z|=1$, and $\nu \geqslant 0$,

$$
\left|w_{\nu}(z)\right| \geqslant \gamma \cdot\left(p_{0}(\nu)-\sum_{0<|i| \leqslant 3}\left|p_{i}(\nu)\right|\right)=\gamma \cdot \sum_{k=0}^{9} \pi_{k} \nu^{k} \geqslant \gamma \pi_{0}=8,297,856,000 \text {. }
$$

It means that (5.3.b) holds. Hence, in particular, we proved that

The orthogonal projectors $Q\left(S_{\xi}^{6 ; 3}\right)$ are uniformly bounded in $L_{\infty}[0,1]$, i.e. $\left\|Q\left(S_{\xi}^{6 ; 3}\right)\right\|_{\infty} \leqslant A<\infty$ for any geometric mesh $\xi$, where $A$ is an absolute constant.

We made all these computations to show how the approach of Sections 1-4 works. One can repeat the analogous computations (from the very beginning) to get the same results for $S_{\xi}^{8 ; 4}$ or $S_{\xi}^{10 ; 5}$ with geometric meshes $\xi$. But it would not give us an understanding of the general situation. What we really need is to consider the following series of conjectures and statements in the case $2^{2 m, m}$.

$$
V^{-1}+S T^{-1} S^{t}=J T^{-1} J \text { and } T+S^{t} V S=J V J
$$

(compare (4.10.a,b)).

(5.8) The polynomial

$$
\begin{aligned}
w_{\nu}(z) & =\operatorname{det}\left\{-\nu S T^{-1} N_{z}+\left(V^{-1}+S T^{-1} S^{t}\right)+\nu N T^{-1} N-N T^{-1} S^{t} \cdot \frac{1}{z}\right\} \\
& =\sum_{i=-m}^{m} P_{i}(\nu) z^{i}=\sum_{i=-m}^{m}\left(\sum_{k=0}^{m} p_{i k} \nu^{k}\right) z^{i}
\end{aligned}
$$

has coefficients such that $p_{0 k}>0,0 \leqslant k \leqslant m^{2}$, and moreover $p_{0 k}>\Sigma_{0<|i| \leqslant m}\left|p_{i k}\right|$. (Compare Tables 1 and 2 or (5.5.1)-(5.5.3).)

(5.9) The coefficients $p_{m^{2} ; k}=0$ if $k \neq 0$ or 1 (it is almost evident), and $p_{m^{2} ; 1}=$ $(-1)^{m-1} \cdot \frac{1}{2} p_{m^{2} ; 0}$ or, equivalently, $p_{0 ;-1}=(-1)^{m-1} \cdot \frac{1}{2} p_{0 ; 0}$; compare (5.4.1)-(5.4.3). (5.8) would give the analog of (5.6) for any $m$. But now we are interested in (5.7)-(5.9) because of the close connections with the theory of Toeplitz block-matrices rather than the analysis of piecewise-polynomial least-squares approximants. 
Later we will consider these statements and conjectures, and other (particular) cases of piecewise-polynomial approximation, in the framework of the approach of Sections 1-4.

Department of Mathematics

The Ohio State University

Columbus, Ohio 43210

1. Z. CiEsIElski, "Properties of the orthonormal Franklin system," Studia Math., v. 23, 1963, pp. 141-157.

2. CARL DE BOOR, "The quasi-interpolant as a tool in elementary polynomial spline theory," in Approximation Theory (G. G. Lorentz, ed.), Academic Press, New York, 1973, pp. 269-276.

3. CARL DE BOOR, "A bound on the $L_{\infty}$-norm of the $L_{2}$-approximation by splines in terms of a global mesh ratio," Math. Comp., v. 30, 1976, pp. 767-771.

4. CARL DE Boor, On a Max-Norm Bound for the Least-Squares Spline Approximant, Conf. on Approximation Theory, Gdansk, Poland, August, 1979. (Preprint.)

5. J. Douglas, JR., T. Dupont \& L. Wahlbin, “Optimal $L_{\infty}$-error estimates for Galerkin approximations to solutions of two-point boundary value problems," Math. Comp., v. 29, 1975, pp. 475-483.

6. S. DEMKo, "Inverses of band matrices and local convergence of spline projections," SIAM J. Numer. Anal., v. 14, 1977, pp. 616-619.

7. S. DemKo, “On bounding $\left\|A^{-1}\right\|_{\infty}$ for banded $A$," Math. Comp., v. 33, 1979, pp. 1283-1288.

8. I. C. Gohberg \& I. A. Feldman, Convolution Equations and Projection Methods for Their Solution, Transl. Math. Monographs, vol. 41, Amer. Math. Soc., Providence, R.I., 1974.

9. H. BART, I. Gohberg \& M. A. KaAshoEK, "Minimal factorization of matrix and operator functions," Operator Theory: Advances and Applications, vol. 1, Birkhäuser Verlag, Basel-Boston-Stuttgart, 1979.

10. B. Mityagin, "Factorization of quasiselfadjoint quadratic pencil," Integral Equations Operator Theory. (To appear.)

11. K. HölliG, " $L_{\infty}$-boundedness of $L_{2}$-projections on splines for a geometric mesh," J. Approx. Theory, v. 33, 1981, pp. 318-333. 